\title{
ANSI/ASTM C-452.75(*) \\ Método de ensayo patrón para medir la expansión potencial de morteros de cemento portland, expuestos a la acción de los sulfatos
}

Traducido y comentado por: RAFAEL TALERo MORALES, Lcdo. en Ciencias Químicas

I.E.T.c.c.

\section{INTRODUCCION}

Este método es aplicable únicamente a cementos portland; el procedimiento se basa en la determinación de la expansión de probetas de mortero hechas con una mezcla de cemento portland y. yeso $\left(\mathrm{SO}_{4} \mathrm{Ca} .2 \mathrm{H}_{2} \mathrm{O}\right)$ en tales proporciones, que la mezcla tenga un contenido de $\mathrm{SO}_{3}$ (trióxido de azufre) del 7,0\% en peso.

Este método es también aplicable ideándose, en primer término, para los interesados en la investigación sobre "métodos para determinar la resistencia potencial del cemento portland a los sulfatos".

\section{APARATOS}

2.1. Balanzas, pesas, probetas de vidrio graduadas, moldes y comparador (fot. 1) de acuerdo con las instrucciones dadas en la norma ANSI/ASTM C 490-77. "Aparatos utilizables para medir los cambios de volumen de la pasta de cemento, mortero u hormigón".

2.2. Mesa de sacudidas, de acuerdo con las instrucciones dadas en la norma ANSI/ASTM C 230-68. "Mesa de sacudidas para los ensayos de conglomerantes hidráulicos".

2.3. Mezclador, cuenco y paleta (fot. 2), según las instrucciones dadas en la norma ANSI/ /ASTM C 305-65.

2.4. Palustre y pisón, de acuerdo con las instrucciones dadas, en la norma ANSI/ASTM C 109-77.

2.5. Cámara húmeda. Se ajustará a las instrucciones dadas en la norma ANSI/ASTM C 511-78.

(*) 1978 ANNUAL BOOK OF ASTM STANDARDS. Part. 13.- “Cement; Lime; Ceilings and Walls; Manual of Cement Testing". Adoptado como Standard el 31-8-64. ASTM Standards. Part. 9 "Cement; Lime; Gypsum". Junio 1965. Designación: ASTM C 452-64. Anteriormente a la adopción de este método como Stanclard se publicó como una simple "tentativa de ensayo" desde 1960 a 1964, habiendo sido revisado en 1963. 


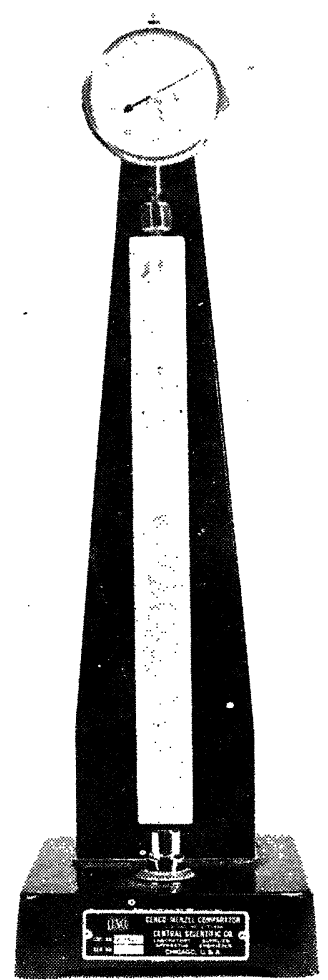

Foto 1.Comparador.

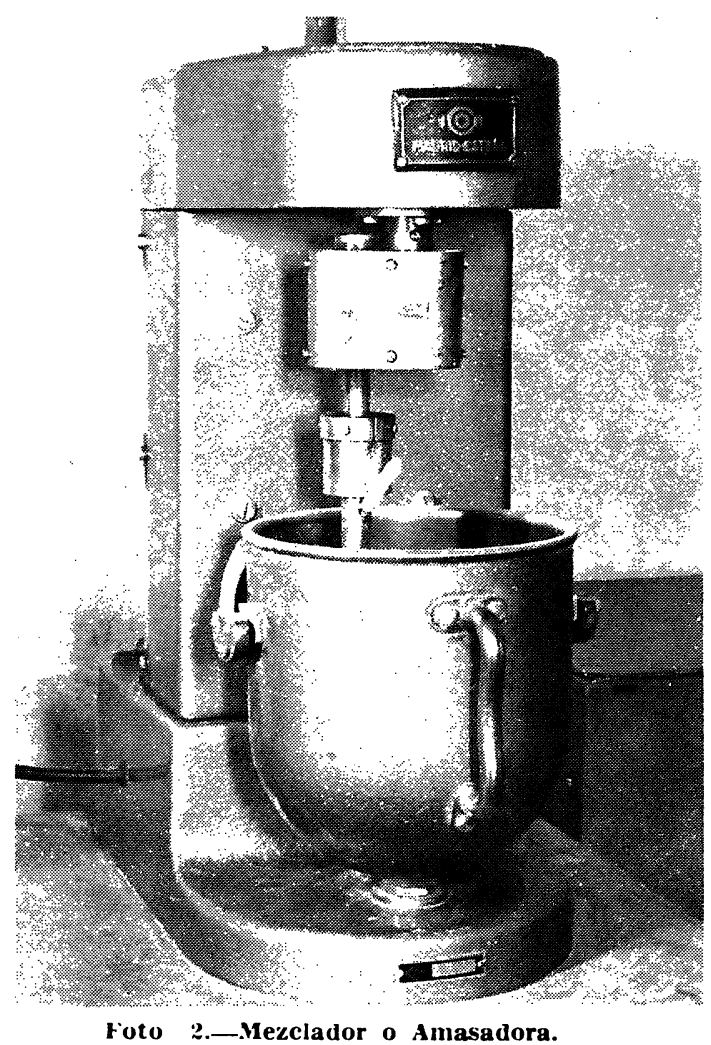

Foto 2.-Mezclador o Amasadora.

\section{TEMPERATURA Y HUMEDAD}

3.1. Cámara de amasado, materiales secos y agua de amasado:

La temperatura de la cámara de amasado, los materiales secos y el agua de amasado se mantendrán entre 20 y $27,5^{\circ} \mathrm{C}\left(68\right.$ y $\left.81,50^{\circ} \mathrm{F}\right)$, y la humedad relativa en la cámara de amasado no será menor del $50 \%$.

\section{MATERIALES}

4.1. La arena normalizada para preparar el mortero con el que confeccionar las probetas de este método de ensayo se acomodará. en todo, a las exigencias expuestas en la sección 3.1. y 4.1. del Método ANSI/ASTM C 109-77.

\begin{tabular}{ccc}
\hline Número de tamiz & Abertura de malla & Retenido (\%) \\
\hline 100 & $150 \mu \mathrm{m}$ & $98 \pm 2$ \\
50 & $300 \mu \mathrm{m}$ & $75 \pm 5$ \\
40 & $425 \mu \mathrm{m}$ & $30 \pm 5$ \\
30 & $600 \mu \mathrm{m}$ & $2 \pm 2$ \\
16 & $1,18 \mathrm{~mm}$ & nada \\
\hline
\end{tabular}

4.2. El yeso utilizado para adicionarlo al cemento portland debe ser yeso natural de gran calidad y cuya tinura de molido ha de ser como sigue:

\begin{tabular}{ccc} 
Número de tamiz & Abertura de malla & Pasante (\%) \\
\hline 100 & $150 \mu \mathrm{m}$ & 100 \\
200 & $75 \mu \mathrm{m}$ & $\geqslant 94$ \\
325 & $45 \mu \mathrm{m}$ & $\geqslant 90$
\end{tabular}


4.3. El contenido de trióxido de azufre $\left(\mathrm{SO}_{3}\right)$ del yeso empleado se deberá obtener utilizando el Método ANSI/ASTM C 471-76. "Análisis químico del yeso y productos yesosos".

4.4. Los porcentajes ponderales de cemento y yeso, para obtener una mezcla de ambos que contenga el $7,0 \%$ de $\mathrm{SO}_{3}$, se han de calcular mediante las siguientes fórmulas:

$$
\begin{gathered}
\text { Cemento, por ciento }(\%)=\frac{g-7,0}{g-c} \times 100 \\
\text { Yeso, por ciento }(\%)=\frac{7,0-c}{g-c} \times 100
\end{gathered}
$$

siendo:

$c=\mathrm{SO}_{3}$ que contiene el cemento portland, en tanto por ciento (\%).

$g=\mathrm{SO}_{3}$ que contiene el yeso, en tanto por ciento (\%).

$7,0=\mathrm{SO}_{3}$ que contiene la mezcla cemento-yeso, en tanto por ciento (\%).

\section{NUMERO Y DIMENSIONES DE LAS PROBETAS DEL ENSAYO}

5.1. Por cada cemento que se ensaye se amasarán dos tongadas de mortero, y de cada una de ellas se prepararán tres prismas (fot. 3) de 25,4 $\times 25,4 \times 285,75 \mathrm{~mm}$ (NOTA 2). Estos prismas son semejantes morfológicamente a las probetas tipo "autoclave" por todos conocidos (fabricantes, usuarios y técnicos del ramo).

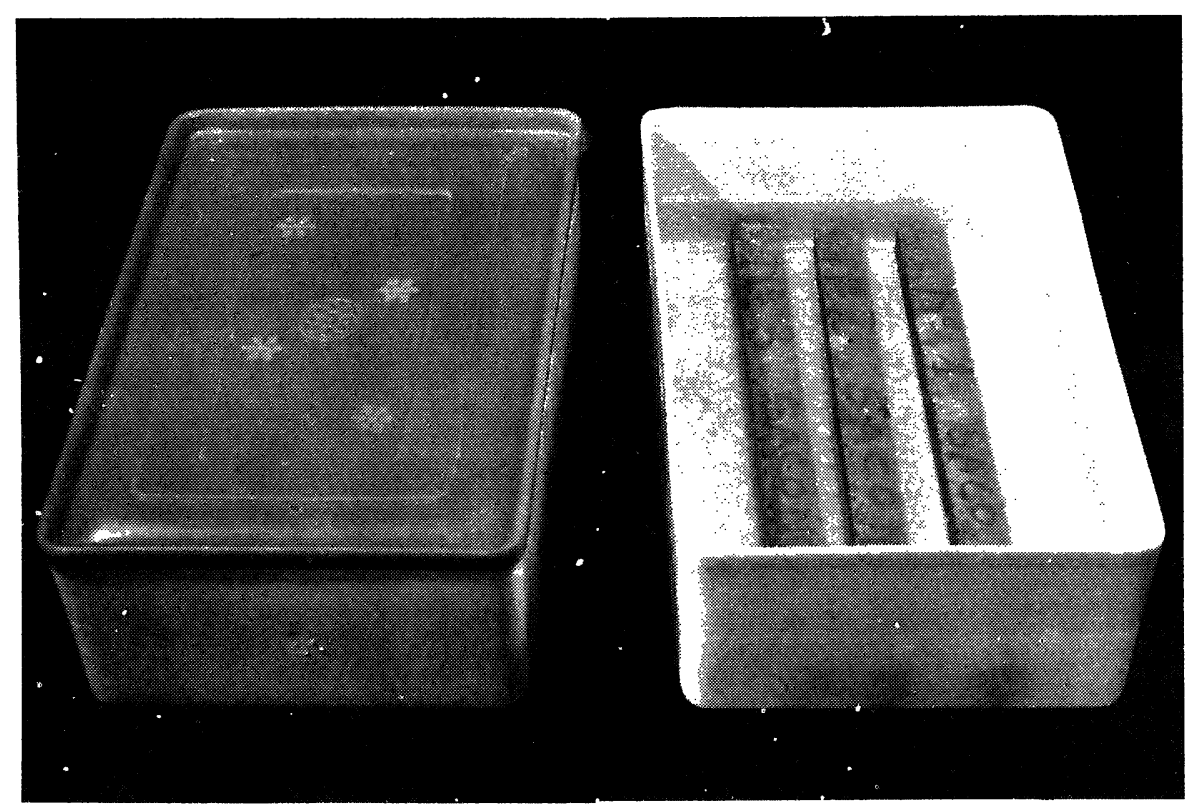

Foto 3.-Aimacenamlento y conservacion de las probetas

NOTA 1.-Ver Anexo.

NOTA 2.-En los ensayos de rutina se pueden utilizar prismas de $25,4 \times 25,4 \times 158,75 \mathrm{~mm}$; en caso de 11 tigio, se usarán los prismas de $25,4 \times 25,4 \times 285,75 \mathrm{~mm}$. 


\section{PREPARACION DE LOS MOLDES}

6.1. Los moldes se prepararán de acuerdo con las condiciones del Método ANSI/ASTM C 490-77, excepto que el interior de los mismos se deberá recubrir con una lámina de plástico o con una ligera capa de aceite de tocino (NOTA 3).

\section{DOSIFICACION, CONSISTENCIA Y MEZCLA DEL MORTERO}

7.1. Las cantidades de materiales secos que se necesitan para cada tongada serán:

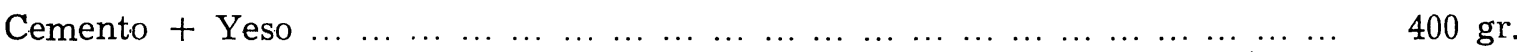

$\begin{array}{llllllllllllllllllllll}\text { Arena normalizada } & \ldots & \ldots & \ldots & \ldots & \ldots & \ldots & \ldots & \ldots & \ldots & \ldots & \ldots & \ldots & \ldots & \ldots & \ldots & \ldots & \ldots & \ldots & \ldots & \ldots & 1.100 \\ \text { gr. }\end{array}$

Agua de amasado:

1.') antes de 1975: el agua de amasado se medía en milímetros de forma que fluya entre 100 y 115, tal y como se determina en la sección 9 del Método ANSI/ASTM C 109-77;

2. ${ }^{\circ}$ después de 1975: $194 \mathrm{ml}$ para todos los cementos portland sin aireantes; $184 \mathrm{ml}$ para todos los cementos portland con aireantes.

7.2. El mortero se mezclará de acuerdo con el procedimiento descrito en la Sección 6 del Método ANSI/ASTM C 305-65 excepto en el punto 2, ya que en la mezcladora primeramente se habrá de añadir el yeso al agua de amasado y, entonces, se mezclan ambos a la misma velocidad lenta durante 15 segundos (140 \pm 5 r.p.m.)

\section{MOLDEO DE LAS PROBETAS DEL ENSAYO}

8.1. Inmediatamente después de la terminación del ensayo de escurrimiento se reintegra el mortero al recipiente, se recoge todo el mortero en el interior del cuenco y se remueve a una velocidad media ( $285 \pm 10$ r.p.m.) durante 15 segundos. Sacar la paleta y el cuenco del mezclador y sacudir el exceso de mortero adherido a la paleta, dentro del cuenco. Llenar el molde en 2 capas, compactando cada capa con el pisón. Colocar el mortero en las esquinas, alrededor del índice y a lo largo de la cara principal del molde, con el pisón hasta conseguir una probeta homogénea. Después que la parte principal de la capa queda compactada, se iguala hasta la altura del molde, y se alisa la cara principal con unos cuantos toques de la espátula.

\section{CONSERVACION De LAS PROBETAS Del ENSAYo}

\subsection{Conservación inicial}

Las probetas se mantendrán en los moldes y en la cámara húmeda a $23 \pm 1,7^{\circ} \mathrm{C}\left(73,4 \pm 3^{\circ} \mathrm{F}\right)$ hasta 30 minutos antes de realizar la medida inicial de su longitud.

\subsection{Almacenamiento posterior}

Una vez que las barras han sido desenmoldadas y medidas se conservarán dispuestas horizontalmente y sumergidas en agua a $23+1,7^{\prime \prime} \mathrm{C}$ (fot. 3). Las probetas se colocarán con una

NOTA 3.-El aceite de tocino es susceptible de inflamarse espontáneainente. Se tendrá un cuidado especial cou el almacenamiento de los trapos usados impregnados con aceite de tocino. 
distancia entre ellas de 6,35 $\mathrm{mm}$ dispuestas en soportes. Las probetas deberán estar cubiertas con una capa de agua de $12,70 \mathrm{~mm}$. La relación entre el volumen de agua y el volumen de las barras no deberá exceder de 5:1 para evitar una excesiva lixiviación. El agua de almacenamiento y conservación deberá rellenarse, con agua fresca, cada 7 días durante los primeros 28 días, y cada 28 días posteriormente.

\section{MEDICION DE LAS LONGITUDES}

a) La longitud de las probetas se medirá mediante el comparador de longitudes (fot. 1). Para ello se sacarán del agua de curado, una a una, y se secarán con un paño antes de realizar la medida.

b) La primera lectura se efectuará a la edad de 24 horas \pm 15 minutos, contados a partir del momento en que el cemento y el agua se hayan puesto en contacto al mezclarlos entre sí. Las probetas se medirán de nuevo a la edad de 14 días (NOTA 4).

\section{CALCULOS}

La diferencia de longitud de las probetas a 24 horas y 7 días se calculará con una aproximación del 0,001\% de su longitud efectiva medida con galga, y se citará como dilatación de la probeta después de ese período de tiempo.

El valor que se anote será la media de por lo menos tres probetas que no difieran entre sí más de $\pm 0,001$ de la media de todas las probetas confeccionadas y ensayadas del mismo cemento.

\section{COMENTARIOS}

Hemos de hacer hincapié tanto en el título del método como en lo expuesto en la INTRODUCCION del presente método de ensayo, donde se exponen con gran claridad y concisión algunos párrafos que, a nuestro juicio, merecen una especial atención, y entre ellos tenemos los siguientes:

TITULO: Método de ensayo patrón ...de morteros, de cemento portland...

INTRODUCCION: Este método es aplicable únicamente a cementos portland;...

Este método es aplicable y se ideó, en primer término, para los interesados en las investigaciones sobre métodos para determinar la resistencia potencial del cemento portland a los sulfatos.

Es decir, en el corto espacio de algunas líneas se repite por 3 veces la aplicación del presente método de ensayo única y exclusivamente a los cementos portland, de donde se deduce el interés que tiene la experimentación, a fin de ampliar si es posible el campo de aplicación del método citado a otros cementos con un contenido total o parcial de fracción portland, tales como los actuales P-Y, PB, PA, PUZ ..., etc. del presente pliego oficial español RC-75, en especial los PA que últimamente y en igualdad de condiciones vienen sustituyendo a los portland en la preparación de hormigones.

NOTA 4.-Como información adicional y una vez obtenidos los valores buscados a las edades de 24 horas y 14 dias, puede introducirse de nuevo las probetas en el agua de conservación o almacenaje a fin de efectuar nuevas medidas a edad (es) posterior (es). 
Los valores tope o límite para diagnosticar a un cemento portland como Sí a No resistentes a los sulfatos, a nuestro juicio deberían estar insertos aquí con tanto o más motivo que en la tabla 2A "Exigencias Físicas discrecionales o Facultativas" del Método ANSI/ASTM C 150-78 [ver tabla 1 columna (I)], por lo que siendo consecuentes con lo que manifestamos aprovechamos la ocasión para exponerlos:

TABLA 1

\begin{tabular}{|c|c|c|c|}
\hline \multirow{2}{*}{ Método ASTMI C 45?-i5 } & \multicolumn{3}{|c|}{$\Delta$ I, Máx. \% } \\
\hline & $I=14$ dias & $\begin{array}{c}\text { II }=28 \text { dias } \\
\begin{array}{c}\text { (nota } \\
6)\end{array}\end{array}$ & $\begin{array}{c}\text { II } \\
(\text { nota } \\
\text { nno }\end{array}$ \\
\hline $\begin{array}{l}\text { Expansión por los sulfatos } \\
\text { (nota 5) }\end{array}$ & 0,045 & 0,059 & 0,164 \\
\hline
\end{tabular}

$\Delta \mathrm{L}=$ Incremento de longitud.

- Cemento portland resistente a sulfatos... expansión $(\%)<0,045$ a 14 días $<0,059$ a 28 días $<0,164$ a 1 año

- Cemento portland no resistente a sulfatos.. expansión (\%) >0,045 a 14 días $>0,059$ a 28 días $>0,164$ a 1 año

Los valores dados a 28 días y 1 año (Tabla 1 , columnas II) están sacados de los Cds. Inv. del IETcc, n. ${ }^{\circ}$ 12, diciembre 1967, pág. 24 y Bull. ASTM n. ${ }^{\circ} 212$, febrero 1965, pág. 135.

De la Asistencia Científico-Técnica realizada en el IETcc y más concretamente en su "División de Durabilidad" se puede deducir que este método, junto con algún otro europeo, son los que más se emplean como exigencias a satisfacer por las diferentes partidas de cemento portland a comerciar en transacciones nacionales e internacionales.

Creemos que éste es un método fácil de preparar en cualquier laboratorio especializado del ramo ya sea regional, provincial, comarcal o local por entender que en todos ellos se deberá disponer de los moldes (similares a los del "ensayo de autoclave" del Pliego Español RC 75), aparatos (amasadora, compactadora, etc.), y posiblemente materiales necesarios para su rápida y sencilla confección y almacenaje.

Asimismo pensamos que éste es un método fácil, universal, rápido (en fabricar probetas, y obtener resultados) y reproducible (ver ASTM Bulletin n. ${ }^{\circ} 212$ february 1956 págs. 37 a 44).

Aunque tanto el título de este método:

METODO DE ENSAYO PATRON PARA MEDIR LA EXPANSION POTENCIAL DE MORTEROS, DE CEMENTO PORTLAND, EXPUESTOS A LA ACCION DE LOS SULFATOS

como la introducción del mismo en su párrafo final:

"métodos para determinar la resistencia potencial del cemento portland a los sulfatos".

NOTA 5.-Cuando se especifique expansión a los sulfatos o por los sulfatos para diagnosticar como si o no sulfato-resistente a un cemento portland, se tendrá ésta en cuenta para realizar tal diagnosis y no los valores límites de $\mathbf{C}_{3} \mathbf{A}$ y $\mathbf{C}_{4} \mathbf{A F}+\mathbf{C}_{3} \mathbf{A}$ dados en la tabla 1 del método ANSI/ASTM C 150-78a.

NOTA 6.-Este método se atiene al resultado obtenido en la expansión de las probetas (en \%) a los 14 dias de edad de las mismas. para definir o diagnosticar como sí o no sulfato-resistente al cemento portland ensayado... 
se refieren a los sulfatos, en general, obviamente y por el tipo de agresivo empleado en este método, $\mathrm{SO}_{4} \mathrm{Ca} .2 \mathrm{H}_{2} \mathrm{O}$ (sulfato cálcico dihidrato o yeso), las conclusiones que de él se extraigan se referirán fundamentalmente a este tipo de los sulfato agresivo, o sea, al cálcico.

Este hecho aunque en principio pudiéranos parecer intrascendente, es o puede ser de particular importancia para aquellas regiones o terrenos con un gran porcentaje de piedra de yeso en su constitución mineralógica como es el caso de España, donde su mitad oriental viene caracterizada de $\mathrm{N}$ a $\mathrm{S}$ por tal constituyente en mayor o menor grado.

Del párrafo n. 11 . CALCULOS, se deduce fácilmente, mediante el límite fijado a las desviaciones de los valores individuales respecto al valor medio, que un incremento del número de probetas llevará consigo una mayor precisión a la hora de facilitar el diagnóstico sobre la posible o no resistencia potencial a los sulfatos de un cemento portland.

A fin de que el juicio a emitir sobre el comportamiento de un cemento portland, según este método de ensayo sea lo más correcto posible, y siempre que se pueda, es aconsejable obtener la expansión porcentual a los 28 días al igual que a los 14 días.

A fin de tratar de subsanar la dificultad que comporta el empleo de arena de Ottawa, ANSI/ASTM C 109. Sec. 3.1., en este método de ensayo se pretende llevar a cabo en este Centro un trabajo de investigación encaminado al tratar de sustituir tal arena por alguna natural de origen nacional (en principio sería deseable la que se emplea para obtener la normalizada del vigente Pliego Español RC-75), de tal modo que los resultados que con ella se obtengan sean estadísticamente equivalentes a los obtenidos con la arena en cuestión especificada por este ensayo ANSI/ASTM C 452-75.

A tenor de lo expuesto en el párrafo final del apartado 1. INTRODUCCION, de este artículo, creemos en la conveniencia de continuar, siempre que sea posible, con los estudios comparativos entre dos o más métodos ya experimentados o no, al objeto de definir o precisar, en su caso, el más idóneo.

\section{A N E X O}

Cemento, por ciento $=\frac{g-7,0}{g-c} \times 100$

$c=\% \mathrm{SO}_{3}$ cemento portland

$g=\% \mathrm{SO}_{3}$ yeso

Yeso, por ciento $=\frac{7,0-c}{g-c} \times 100$

$7,0=\% \mathrm{SO}_{3}$ mezcla cemento-yeso

Estas fórmulas aparentemente mágicas y misteriosas, al no citar este método su origen a procedencia, podemos decir, sin ningún género de dudas, que son el resultado final de un simple ejercicio o problema de mezclas, o mejor, dosificaciones adecuadas para preparar una determinada mezcla, de cemento y yeso, que es el caso que nos ocupa. De este tipo de problemas de mezclas, el libro "Manuales y Normas del IETcc. Cemento Portland. Cálculo e Interpretación de datos químicos": del Prof. Dr. D. José Calleja Carrete, posee, en el apartado 3.2. dedicado a "Dosificación", págs. 105 a 109 un ejemplo patrón (además de algún otro de tipo práctico) sobre la resolución de este tipo de problemas que, a nuestro juicio, ha sido tratado, expuesto y resuelto por el autor con gran acierto y claridad, y que con el permiso verbal del mismo nos hemos permitido reproducir adecuando 
cada letra, signo o símbolo correspondiente con su significado respectivo para nuestro caso, cemento y yeso. Ateniéndonos, como decimos, a los signos, y su significado respectivo, expuestos en el encabezamiento de este ANEXO tenemos que:

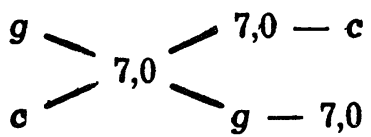

donde como sabemos:

$$
\begin{aligned}
c & =\% \mathrm{SO}_{3} \text { cemento portland a ensayar, } \\
g & =\% \mathrm{SO}_{3} \text { yeso, } \\
7,0 & =\% \mathrm{SO}_{3} \text { de la mezcla cemento más yeso, }
\end{aligned}
$$

o sea, como vemos, se disponen los datos numéricos (obtenidos previamente por la vía química analítica cuantitativa, correspondiente); en forma de aspa, de manera que:

- en la parte superior izquierda queda el tanto por ciento de $\mathrm{SO}_{3}$ del yeso $(g)$;

- en la parte inferior izquienda queda el tanto por ciento de $\mathrm{SO}_{3}$ del cemento portland (c);

- en el centro del aspa, queda el tanto por ciento que se desea que contenga la mezcla a obtener de cemento más yeso, que en este caso es 7,0;

- se calculan las diferencias $7,0-c$ y $g-7,0$, que se situarán respectivamente:

- 7,0 - c en la parte superior derecha;

- $g-7,0$ en la parte inferior derecha;

- su cociente, en el orden en que quedan situadas, o sea, $\frac{7,0-c}{g-7,0}$ es igual a la razón o proporción de mezcla $y / x(y=$ partes en peso de componente yeso; $x=$ partes en peso de componentes cemento) con que deben formar parte de la mezcla, cemento más yeso, para que ésta cumpla la condición de tener por valor de $\mathrm{SO}_{3}$ el 7,0 por ciento. Si la cantidad a preparar de dicha mezcla es, por ejemplo, $100 \mathrm{gr}$ :

$y=$ gr. de yeso en $100 \mathrm{gr}$. de mezcla cemento más yeso del $7,0 \%$ de $\mathrm{SO}_{3}$,

$x=$ gr. de cemento en $100 \mathrm{gr}$. de mezcla cemento más yeso del $7,0 \% \mathrm{SO}_{3}$.

La resolución del problema ya se nos presenta bastante fácil, pues hemos desembocado en un sistema de dos ecuacioes:

$\begin{aligned} & \frac{y}{x}=\frac{7,0-c}{g-7,0} \\ & y+x=100\end{aligned} \mid$ con dos incógnitas $x$ e $y$ que resuelto nos da $\mid \begin{aligned} & y=\frac{7,0-c}{g-c} \times 100 \\ & x=\frac{g-7,0}{g-c} \times 100\end{aligned}$

fórmulas éstas que como vemos coinciden, en todo, con las "bautizadas" anteriormente como "aparentemente mágicas y misteriosas"dadas por el método en cuestión ANSI/ASTM C-452-75 en su apartado 4.4. 